\title{
Mitteilungen der MKÖ
}

J. Urol. Urogynäkol. AT 2020 • 27:144

https://doi.org/10.1007/s41972-020-00125-3

c Springer-Verlag GmbH Austria, ein Teil von Springer Nature 2020

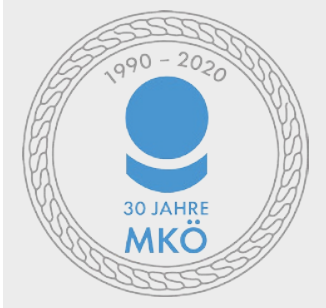

Präsidentin

OÄ Dr. Michaela Lechner, FEBS (V.i.S.d.P.)

MKÖ

Schwarzspanierstraße 15/3/1

A-1090 Wien

Tel. +43(0)1-4020928

info@kontinenzgesellschaft.at

\section{Online-Symposium: Der schmerzhafte Beckenboden}

Anstelle der geplanten Jubiläumstagung der Medizinischen Kontinenzgesellschaft Österreich (MKÖ) öffnete die Gesellschaft heuer Corona-bedingt erstmals ihre virtuellen Türen. Das Online-Seminar widmete sich dem schmerzhaften Beckenboden, der meistens multifaktoriell bedingt ist und daher multidisziplinär betrachtet werden muss. Das setzt eine gute Vernetzung aller beteiligten Fachgruppen voraus, was seit nunmehr 30 Jahren ein erklärtes Ziel der MKÖ ist.

Zwölf Vortragende aus verschiedenen Fachdisziplinen beleuchteten im Rahmen des vierstündigen Intensiv-Webinars ihren Zugang in den unterschiedlichen Facetten. In den Vorträgen wurden die vielfältigen Ursachen aber auch spezielle Aspekte des chronischen Beckenschmerzes wie Sexualfunktionsstörungen oder das Pelvic Congestion-Syndrome (PCS) behandelt. Darüber hinaus wurde versucht, dem Mysterium des Symptomenkomplexes „Bladder Pain Syndrome“ (früher oft auch interstitielle Zystitis) auf die Spur zu kommen.

„Die dreistellige Teilnehmeranzahl bereits in der ersten Stunde zeigte, wie relevant das Thema und wie groß der Wunsch nach Austausch ist", freuten sich die beiden Tagungspräsidenten Katharina Meller, PT und Priv. Doz. Dr. Nikolaus Veit-Rubin über das rege Interesse. „Mit der virtuellen Jahrestagung haben wir einen Schritt in Richtung

\section{Save the date}

Die 30./31. Jahrestagung der MKÖ findet am 15. und 16. Oktober 2021 (hoffentlich) wieder im LFI Linz auf der Gugl statt.

Digitalisierung gewagt und sind zuversichtlich, dass uns dies für die Zukunft viele neue Möglichkeiten eröffnet. Unsere Motivation und den Innovationsgeist der MKÖ nehmen wir für 2021 mit nach Linz und hoffen dort auf eine Tagung, bei der auch der persönliche Austausch wieder möglich ist!“

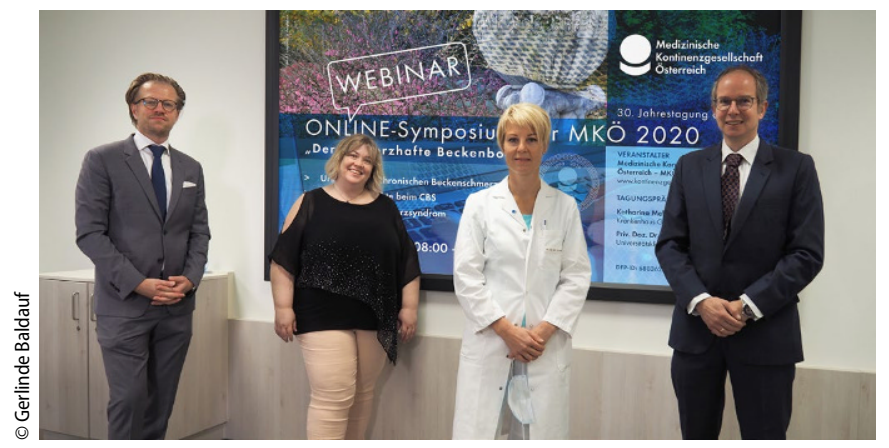

$\Delta$ v. I.n.r. Priv. Doz. Dr. Nikolaus Veit-Rubin, Katharina Meller, PT, OÄ Dr. Michaela Lechner (MKÖ-Präsidentin), OA Dr. Michael Rutkowski (MKÖ-Vizepräsident) 